\title{
Coordinating epistemic frames in informal physics: Agency, support, and technology
}

\author{
Brett L. Fiedler and Michael B. Bennett \\ JILA NSF Physics Frontier Center, University of Colorado Boulder, 440 UCB, Boulder, CO, 80309 and \\ Department of Physics, University of Colorado at Boulder, 390 UCB, Boulder, CO, 80309
}

Nicole E. Johnson

ATLAS Institute, University of Colorado Boulder, 320 UCB, Boulder, CO, 80309

Emily B. Moore

Department of Physics, University of Colorado Boulder, 390 UCB, Boulder, CO, 80309

\begin{abstract}
Informal physics environments present opportunities for youth to voluntarily engage with physics in a collaborative space. However, individual children's educational backgrounds, expectations, and motivations impact their engagement with the group learning activities and educational tools in informal physics learning. We initiate an investigation into the variables of agency, support, and technology that contribute to children's dynamic epistemic framing of a virtually simulated learning environment within an afterschool physics environment. Specifically, we consider the use of PhET interactive simulations in the PISEC afterschool physics program by middle-school students and their college-aged mentors. We analyzed video of a group of three learners and their mentor as they use a simulation and transition into a physical experiment based on the simulation. We analyzed each individual's actions and vocalizations to identify their framing using a two-dimensional framing axis. Further, we determined when these frames appeared to be aligned or misaligned between group members during the activity, transitions between alignment and misalignment, and investigated what malleable factors (e.g. technology use, mentor pedagogy, program structures) are contributing to these shifts in alignment. Initial analysis shows malleable factors such as UE pedagogy and environment affordances (e.g. technology, spatial orientation), as well as their intersection with non-malleable factors (e.g. youth social motivations) for future investigation.
\end{abstract}

2019 PERC Proceedings edited by Cao, Wolf, and Bennett; Peer-reviewed, doi.org/10.1119/perc.2019.pr.Fiedler Published by the American Association of Physics Teachers under a Creative Commons Attribution 4.0 license. Further distribution must maintain attribution to the article's authors, cover page, and DOI. 


\section{INTRODUCTION}

Informal physics environments provide a unique opportunity to study student-directed learning. Participants in informal physics environments bring a diversity of content knowledge, pedagogical expectations, and motivations to the learning activity. When engaged in group learning activities in informal settings, the diversity of participants' ways of engaging can significantly impact the experience of the activity for all group members. For those creating, directing, or facilitating informal physics learning experiences, it can be difficult to disentangle the impact of individual participants, program structure, and the physics activities involved to understand what malleable factors are contributing to more or less positive experiences for learners. In this work we focus our investigation on groups engaged in simulation-based activities as part of an established informal physics program. Our overarching research question is: How do malleable program factors (e.g., mentor facilitation, activity structure, technology use) contribute to alignment or misalignment between group participants' framing in open-ended informal physics learning environments?

To begin to address this question, we build upon prior research identifying learner's epistemic frames conducted within formal settings. Framing is a groups' or individuals' interpretation of a situation and how they should act within it [1]. Epistemic framing specifically attends to a student's sense of what is occurring and how to act within a learning situation. Research on epistemic framing within the resources framework [2] has shown it to be dynamic, highly dependent on the context, and transferable across domains [3-6]. Prior work has successfully leveraged epistemic framing studies to investigate student behaviors when engaging in formal contexts in small groups (e.g., [5]).

Each student may activate different resources depending on where they perceive a situation to be across the informal to formal continuum [7]. Prior research has analyzed the framing of collaborative groups, utilizing the group as the level of analysis. Within our context, an informal afterschool program, each child brings different expectations to the program activities. During an activity, the participants must determine (and co-create) the nature of the activity together, each bringing a unique set of prior experiences that help shape their framing of a situation. The alignment of these individual's frames, the extent to which children share expectations for their learning activity, has the potential to enhance or hinder the learning experience for the children involved.

To investigate mediating factors that influence alignment between individual learner frames in informal physics programs, we apply a 2-axis framing framework [8] to data collected during an afterschool physics education program, the Partnerships for Informal Science Education in the Community (PISEC). The collected data involves middle school children and college-aged mentors engaged in simulation-based (PhET Interactive Simulations) learning activities.

We present an initial investigation of each participants' epistemic framing during a group-selected simulation-based activity, identifying significant frame shifts. We then analyze the factors that contribute to group frame alignment or misalignment at the intersection of agency, support, and technology. This study aims to advance research tools to study and iterate on informal physics programs in line with designbased research goals.

\section{STUDY DESIGN}

\section{A. Context}

Data was collected during learner participation in the PISEC program, which is a joint collaboration between the JILA NSF Physics Frontier Center and the PER group in the physics department at the University of Colorado Boulder [9]. PISEC is a physics program rooted in the Fifth Dimension afterschool model [10]. The Fifth Dimension model emphasizes youth agency while advancing rich learning experiences, the use of technology to engage children's interest, and collaboration amongst groups of children and adults with diverse backgrounds and life experience. PISEC operates afterschool programs that bring together university-affiliated volunteers (University Educators or "UEs") with underrepresented minority learners in weekly sessions for 8-10 weeks each semester. During these sessions, UEs work with groups of 2-5 students with hands-on and simulation-based physics activities from a set of designed curricula (e.g. Electricity and Magnetism).

Aligned with the child agency and technology focus of the Fifth Dimension model, PhET Interactive Simulations (PhET sims) [11] have been incorporated into the PISEC program and are part of multiple activities learners have to choose from in each curriculum set. Because of the informal structure and child agency focus of PISEC, learners may choose to engage in an activity to the extent they desire according to their own goals. Each group decides which activity to select, when to end an activity, and how to engage with an activity. Each learner has a PISEC notebook to plan their experiment and record their findings. A PISEC activity typically consists of a planning period, an experimenting period, and a recording period, however, the order, duration or presence of these is decided by the group and/or individuals.

Here, we focus on data collected recently from the PISEC afterschool setting in which a group of three 7 th grade children and a UE were participating in the Electricity and Magnetism curriculum. In this setting, 5 out of 27 of the activities children could choose from included sim-based activities, utilizing PhET sims accessed on a set of 6 tablet devices (iPads).

On this particular day, the group chose the sim activity John Travoltage [12], accompanied by a short prompt [13]. With this sim, learners can explore the concept of static electricity by rubbing John's foot on a rug (transferring negative charges onto his body) and then moving his arm to discharge the charges through his hand into a nearby doorknob - with a 

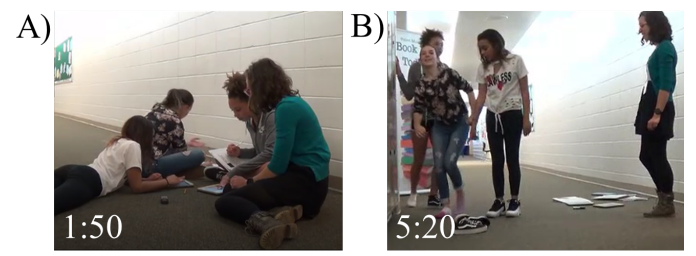

We use the two-dimensional framing axis (expansive vs. narrow along one axis and silly vs. serious along the other) as developed by Irving [8]. This framework leverages epistemic frames through the resources framework [2] to account for flexibility in student reasoning. A narrow framing focuses on ideas related to the problem at hand, in contrast to an expansive framing that focuses on the generalizability of an idea [14]. Silly framing focuses on aspects of joking and play, while serious framing focuses on straightforward activity progression. Notably, within this framework frames are not discrete, but are considered to exist along a continuum.

\section{Methods}

Data collection and post-processing. Audio and video are routinely collected at every site in the PISEC afterschool program. During the last year, audio and video collection emphasized groups opting to work on sim-based activities. Since every activity is chosen at will by the groups during each 1-hour session, cameras and audio recorders are re-positioned by onsite research staff as needed and screen capture software is started on the devices when in use. To aid syncing of the audio, camera and screencapture video data collected, a loud auditory cue is made during recording. In post-processing, video editing software is used to sync each group's video and screen capture files, generating a single multi-split screen video file with clear audio showing all recorded sim interaction for analysis.

Analysis. We conducted video analysis of gaze, body language, sim interaction, and dialog characteristics (synchronicity, topic, direction, volume/tone) during the John Travoltage sim activity. The entire activity lasted 10 minutes. At 4 minutes 17 seconds (4:17), the group transitions from sim use to spontaneous physical experimentation. We present analysis of the first 6 minutes of the activity, inclusive of the sim-focused component and their transition away from sim interaction.

We focused analysis on behaviors existing within an 'activity frame' when individuals' speech and/or actions are directed toward the activity, rather than a 'non-activity frame' when their speech and/or actions reflect other aspects of the afterschool environment (e.g. social talk or off-topic technology use). The axes of the frame plots are normalized to the context of PISEC and sim use; plot points indicate relative framing of individual group members.

We began by mapping the behaviors identified in Irving et al. [8] for expansive/narrow and silly/serious framing as a baseline and incorporated simulation-specific behaviors and speech aspects. For example, we consider the following behaviors in the narrow half-axis: superficial focus on the sim
FIG. 1. Group arrangement during A) simulation activity and B) physical enactment of the sim.

display (character appearance or audio) or interactions (moving hand or foot); focus on activity prompt; or exclusively following UE recommendations. We consider the following behaviors in the expansive half-axis: learner focuses on the concept of static electricity, uses real-world connections, gestures as explanation and connects to hands-on activities. Behaviors indicative of silly framing include: laughter after statements, sarcastic tone of voice, and physical play.

We then identified behavioral clusters according to observations categorized along the two axes. Initial practice plots were generated by two researchers and discussed with respect to a small example clip unrelated to the case study to familiarize researchers with behaviors typical of each category (expansive/narrow and silly/serious). The case study presented was independently coded by two researchers, followed by discussion to resolve differences. There was good agreement on quadrant assignment, with differences arising solely from moments of ambiguous learner verbalizations.

\section{FRAMING DURING SIMULATION ACTIVITY}

The activity starts (0:00) with the children sitting on the floor in a hallway (Fig. 1A), opening the sim on their individual iPads. While there is conversation amongst group members and the mentor as the activity progresses, each learners' inquiry with the sim follows an independent path. At 4:18, the UE asks a question about their experience with static electricity, which leads to the group working together in an attempt to create a static shock in real life by rubbing their socks on the carpet (Fig. 1B). Figure 2 shows the epistemic frame plots generated for each student and the UE.

Blanc (Fig. 2A) is the first to begin interacting with the sim. She begins by commenting $(1: 19, \mathrm{Na}-\mathrm{Si})$ on the "weird" appearance of John. She begins to experiment, building up charge, observing a discharge, and then verbalizes plans to build up a large amount of charge. She does this, then causes a discharge. She reports this to the group, but receives no response (2:13, Ex-Se). After the UE asks "what is happening?", she identifies (first in the group) the concept, "static electricity" (2:51, Ex-Si). Her shifts toward a more expansive framing of the activity occur during sim interaction and interaction with the UE. Blanc spends most of the activity along the Silly axis and we consider her to be a strong influencer for the rest of the group, including the UE. During the activ- 

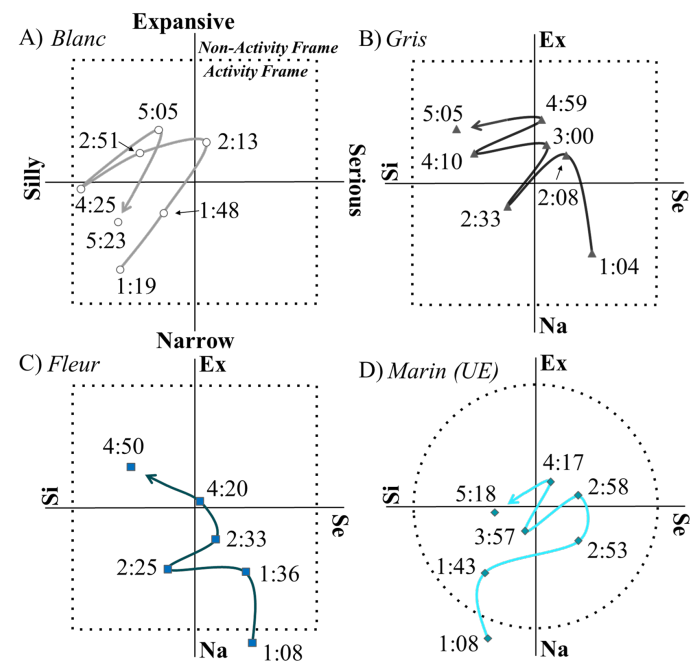

FIG. 2. Frame plots for the learners (Blanc, Gris and Fleur) and the $\mathrm{UE}($ Marin) along expansive $(\mathrm{Ex})$ or narrow $(\mathrm{Na})$ and silly $(\mathrm{Si})$ or serious (Se) framing axes.

ity, she frequently uses a playful tone when interacting with her peers and engages in physical play ('rough-housing') with the other children. She appears to view the PISEC program as an opportunity to have fun with her friends while doing the science experiments.

Gris (Fig. 2B) begins by writing in the planning section of her notebook. Gris tells the group she has only written that she will "play" with the sim (1:04, Na-Se), unsurprising as they have not interacted with this sim before and do not know what they will be able to test. Gris begins playing with the sim and announces "Oh, he can kick stuff!". At 2:08 (Ex-Se), she looks at Blanc's iPad where charges are building up when John's foot is rubbed on the carpet. She begins to verbalize what she sees, interrupts herself ("Hold on. Wait") and begins to interact with her iPad, possibly testing an idea she had. She tries (unsuccessfully) to share with her group what she found. Gris is pulled over into the silly axis at both 2:33 (Na$\mathrm{Si})$ and 4:10 (Ex-Si) by jokes from Blanc, which turned into a playful challenge from Gris as to the veracity of Blanc's claims (e.g. "[giggling] No...that isn't right."). After each of these more Silly exchanges, she returns to her notebook and the sim. Gris does not show shifts in response to the UE and is most influenced by peer interactions (Silly) and notebook or sim interactions (Expansive-Serious).

Fleur (Fig. 2C) has limited interaction with most of the group, not shifting outside of a narrow axis during her sim use. Fleur has difficulty getting the sim started on her iPad and is turned away from the group toward her iPad (1:08). Fleur looks at her peer's iPads when she is unable to access her own but does not engage in any discussion and writes in her notebook while she waits for her own iPad to be set up (1:36, Na-Se). When the sim starts, she focuses on the movement she can do with John's leg, which she announces to the group, with no response $(2: 25, \mathrm{Na}-\mathrm{Si})$. Fleur purposefully shocks John and makes a second bid for group interaction, targeted at Gris: "Oh my god, watch this guys! Guys! Watch this! [Gris], watch this! [Gris]...Watch this! [Gris]! Watch this!", without response. Despite no indicators of expansive framing within the sim activity, Fleur is the first to respond to the UEs question (4:20, Ex-Se) regarding their real-world experience with static electricity and initiates a new investigation involving physically experimenting by rubbing their feet on the carpet to create a shock (and later, her head (4:50)) which we consider to be an expansive activity.

The UE, Marin (Fig. 2D), is included in this analysis within PISEC UEs are an integral part of the learner group and are not restricted to any particular mode of interaction with the learners. From our observations, UEs engage with the student activities using a broad range of frames and pedagogical approaches [15]. Regardless of a UE's individual frame, we also recognize that collectively UEs are inherently engaged in a different mentor-type of activity than the learners and do not include them in the alignment analysis. The circle as the Activity Frame in Fig. 2D represents this difference. However, we analyze the behavior of the UE using the same framework as the learners to illuminate clues into how the UE adjusts their framing of the activity and modulate their own behaviors in order to engage the students. For example, at 1:43, Marin laughs and agrees with Blanc that John looks "unnatural". This appears to be an attempt by Marin to integrate herself into this group of friends, while simultaneously bringing herself into the activity that Blanc is doing. By mimicking Blanc, Marin can make inroads to ask more questions about what is happening in the sim toward more expansive behaviors.

\section{FRAME ALIGNMENT DURING SIM ACTIVITY}

Using the framing plots generated for each student, we can identify overlaps (or lack thereof) in framing between members of the group and investigate potential influencing factors. In our analysis of this group's activity, we determine that while there is occasional overlap in framing between members of the group, their verbalizations and actions indicate they are rarely in coordination with one another - until they transition to and engage in the physical experiment at 4:20 (Fig. 3).

During the initial sim interactions (1:04-2:07) each child obtained their own device and began the sim at different times. As highlighted in Fig. 3A, Gris and Fleur overlap in the Narrow-Serious frame, but are not interacting with each other. Blanc stays within another frame (Na-Si). Fleur does not have her sim open, while the others have begun their sim play. Fleur is positioned facing away from the other members of the group and each student is looking down at their device. When they speak, they often speak to the device and not to any particular group member. The UE is present and assisting in technical support to get the sim open for all of the learners. However, she does not introduce the concept underly- 


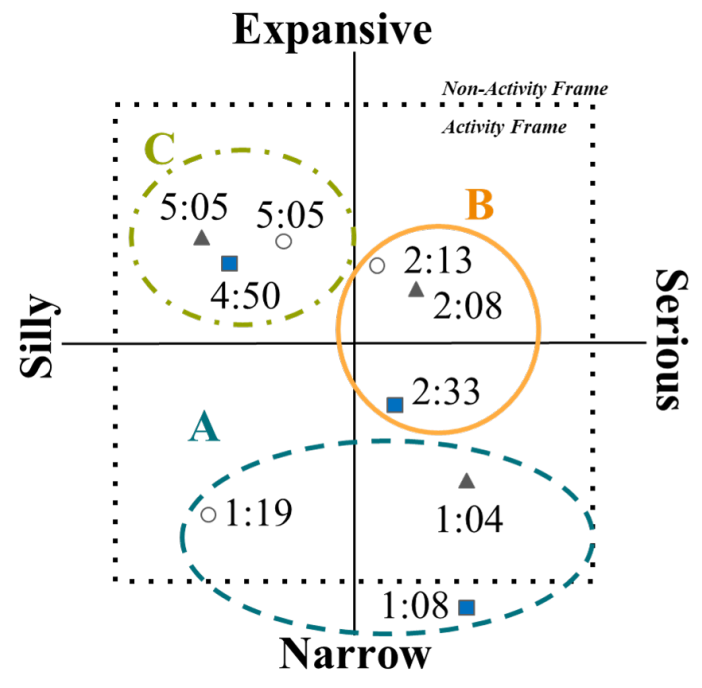

FIG. 3. Frame alignment of Blanc (circle), Gris (triangle) and Fleur (square) highlighted at three periods (A, B and C) during the group activity.

ing the sim and waits until they explore to begin questioning, addressing each child individually and not the entire group. We think the nature of their spatial orientation (Fig. 1A), 1:1 access to iPads, and lack of a synchronous sim start results in the group being physically close in proximity but lacking a shared referent, contributing to a lack of collaborative engagement.

From 2:08-4:19, Blanc and Gris overlap in the ExpansiveSerious frame (Fig. 3B), with occasional comments toward each other without reciprocation. However, we see that Fleur is still engaged in a narrow framing while the other group members have begun making conceptual connections using the sim. Fleur demonstrates a similar progression through the activity as the other learners, making similar discoveries as the other group members, but about a minute later in time. When she shares her discoveries, she is met with no response from the rest of the group. In fact, this occurs for each student in this time segment. Gris tries to get Fleur's attention to share that she had shocked John with the maximum possible charge. Blanc tries to get the groups' attention when she maximizes the charge build-up in John also, but receives no feedback. This appears to be a pivotal moment for Blanc and the only time she appears to frame this as a serious learning activity. When she receives no response, she asks "what's the point of this stupid game?". While Fleur never aligns with the others in expansive framing during the sim activity, Blanc and Gris progress at similar rates in the sim nearby the UE and thus align in expansive framing.

Interestingly, even in the absence of alignment of the group's framing, there is collective group movement from narrow toward expansive framing over the duration of sim use, with occasional pairwise learner and UE engagement. The rapid transition to a collective group physical experiment at 4:20 - also a transition to a group-alignment of framing, is indicative of the children's social motivations within the afterschool program [16].

At 4:20, the UE asks: "Have you guys tried this yourselves?" to which Fleur raises her gaze to the UE, smiles, and responds: "Should I?". At this point, all of the learners begin to converge in aligned framing (Fig. 3C). Fleur enters the Expansive-Silly frame, when the other group members join her in generating ideas and enacting physical experiments at 5:05. Fleur stands up and attempts to rub her shoe on the ground, which initiates a discussion with all members in the group regarding the potential effect of the rubber. The the children stand up, face each other, first trying to emulate John in the sim and then expanding to determine what they need to do to elicit a shock from their peers (Fig. 1B). From then until they leave the camera view, the group engages in coordinated discussion and action.

The group coordination present in the physical activity is supported by the flexibility and agency afforded by the PISEC program, in which students are not bound to a single scripted experiment, but rather are free to build on the provided material to explore their curiosity. The trajectory of their activity was significantly influenced by their interactions with the sim, even when the group transitioned away from sim use. Additionally, we speculate that their adherence to a silly frame serves to create a space which enables all members the comfort to participate, as Marin did in her interactions with Blanc early in the activity. As in formal settings, silly framing can be a powerful tool for creating safe spaces for students to suggest ideas without rebuke [8, 17].

\section{v. CONCLUSIONS}

The case study presented exemplifies some of the complex, intersecting factors that contribute to a coordinated group learning experience. Analysis indicates key malleable factors for further investigation to promote group coordination, such as UE pedagogy and environment affordances (e.g. technology, spatial orientation), as well as their intersection with non-malleable factors (e.g. youth social motivations). This type of analysis within an informal setting also presents a unique opportunity to explore the requirements of novel technology to promote group coordination in student-directed learning contexts. Our analysis of the UE within the same framework as the students presents a potential approach for future studies of teaching strategies through framing analysis in informal settings. We will explore these and other factors further during analysis of the full data set.

\section{ACKNOWLEDGEMENTS}

We thank all participants in the PISEC program. This material is based on work supported by the National Science Foundation DRL-\#1621363, PHY-\#1734006, and DMR\#1548924. 
[1] D. Tannen, Framing in Discourse (Oxford University Press, New York, 1993).

[2] D. Hammer, E. F. Redish, A. Elby, and R. E. Scherr, Resources, framing, and transfer, in Transfer of Learning: Research and Perspectives, edited by J. Mestre (Information Age Publishing, Charlotte, NC, 2004).

[3] Nguyen, H. D., Chari, D. N. and Sayre, E. C. Dynamics of students' epistemological framing in group problem solving. Eur. J. Phys. 37, 065706 (2016).

[4] Rosenberg, S., Hammer, D. and Phelan, J. Multiple Epistemological Coherences in an Eighth-Grade Discussion of the Rock Cycle. J. Learn. Sci. 15, 261-292 (2006).

[5] Scherr, R. E. and Hammer, D. Student behavior and epistemological framing: Examples from collaborative active-learning activities in physics. Cogn. Instr. 27, 147-174 (2009).

[6] Shaffer, D. W. Epistemic frames for epistemic games. Comput. Educ. 46, 223-234 (2006).

[7] National Research Council, Learning Science in Informal Environments: People, Places, and Pursuits (National Academies Press, Washington, DC, 2009).

[8] Irving, P. W., Martinuk, M. S. and Sayre, E. C. Transitions in students' epistemic framing along two axes. Phys. Rev. Spec. Top. -Phys. Educ. Res. 9, 1-11 (2013).
[9] Partnerships for Informal Science Education in the Community, http://www.colorado.edu/physics/PISEC/

[10] M. Cole, The Fifth Dimension: An After-School Program Built on Diversity (Russell Sage Foundation, New York, 2006).

[11] PhET Interactive Simulations, http://phet.colorado.edu/

[12] John Travoltage PhET Interactive Simulation, https://phet. colorado.edu/en/simulation/john-travoltage

[13] Activity prompt used by students in the simulation activity located at https://www.colorado.edu/outreach/pisec/sites/default/ files/attached-files/johntravoltage_activityprompt.pdf

[14] Engle, R. A., Lam, D. P., Meyer, X. S. and Nix, S. E. How Does Expansive Framing Promote Transfer? Several Proposed Explanations and a Research Agenda for Investigating Them. Educ. Psychol. 47, 215-231 (2012).

[15] Hinko, K. A., Madigan, P., Miller, E. and Finkelstein, N. D. Characterizing pedagogical practices of university physics students in informal learning environments. Phys. Rev. Phys. Educ. Res. 12, 010111 (2016).

[16] Fiedler, B. L., Fracchiolla, C., Bennett, M. B., Hinko, K. A., and Finkelstein, N. A Design-Based Informal Physics Program from a Youth Perspective, presented at the Physics Education Research Conference 2018, Washington, DC, 2018

[17] G. Bateson, A Theory of Play and Fantasy (Ballantine, New York, 1972). 